九州大学学術情報リポジトリ

Kyushu University Institutional Repository

\title{
A methodological and preliminary study on the acoustic effect of a trumpet player's vocal tract
}

Kaburagi, Tokihiko

Faculty of Design, Kyushu University

Yamada, Naoyuki

Graduate School of Design, Kyushu University

Fukui, Takashi

Shikumi Design Company, Ltd.

Minamiya, Eriko

Yamaha Corporation

http://hdl. handle. net/2324/25703

出版情報: Journal of the Acoustical Society of America. 130 (1)，pp.536-545，2011-07. Acoustical Society of America

バージョン:

権利関係 : (C) 2011 Acoustical Society of America 


\title{
A methodological and preliminary study on the acoustic effect of a trumpet player's vocal tract
}

\author{
Tokihiko Kaburagia) \\ Faculty of Design, Kyushu University, 4-9-1 Shiobaru, Minami-ku, Fukuoka, 815-8540 Japan \\ Naoyuki Yamada \\ Graduate School of Design, Kyushu University, 4-9-1 Shiobaru, Minami-ku, Fukuoka, 815-8540 Japan \\ Takashi Fukui \\ Shikumi Design Company, Ltd. Hakata Hojo Building 401, 4-8-15 Hakata-Ekimae, Hakata-ku, Fukuoka, \\ 812-0011 Japan \\ Eriko Minamiya \\ Yamaha Corporation, 10-1 Nakazawa-cho, Naka-ku, Hamamatsu, Shizuoka, 430-8650 Japan
}

(Received 3 February 2011; revised 6 May 2011; accepted 9 May 2011)

\begin{abstract}
A methodological study is presented to examine the acoustic role of the vocal tract in playing the trumpet. Preliminary results obtained for one professional player are also shown to demonstrate the effectiveness of the method. Images of the vocal tract with a resolution of $0.5 \mathrm{~mm}(2 \mathrm{~mm}$ in thickness) were recorded with magnetic resonance imaging to observe the tongue posture and estimate the vocal-tract area function during actual performance. The input impedance was then calculated for the player's air column including both the supra- and subglottal tracts using an acoustic tube model including the effect of wall losses. Finally, a time-domain blowing simulation by Adachi and Sato [J. Acoust. Soc. Am. 99, 1200-1209 (1996)] was performed with a model of the lips. In this simulation, the oscillating frequency of the lips was slightly affected by using different shapes of the vocal tract measured for the player. In particular, when the natural frequency of the lips was gradually increased, the transition to the higher mode occurred at different frequencies for different vocal-tract shapes. Furthermore, simulation results showed that the minimum blowing pressure required to attain the lip oscillation can be reduced by adjusting the vocal-tract shape properly.
\end{abstract}

(C) 2011 Acoustical Society of America. [DOI: 10.1121/1.3596471]

PACS number(s): 43.75.Fg [NHF]

Pages: 536-545

\section{INTRODUCTION}

The mechanism of sound generation by brass instruments has been mainly investigated with regard to the resonance characteristics of the instrument bore and self-oscillating mechanism of the lips. The resonance frequency of the instrument strongly affects the lip motion and usually determines the blowing pitch. The input impedance was therefore measured by Backus ${ }^{1}$ for various instruments. Nonlinear wave propagation in the bore is thought to be important to brass instruments in that it characterizes the specific timbre, and thus, it has also been studied. ${ }^{2-4}$ In the case of lip motion, the condition for obtaining self-oscillation was theoretically derived by Fletcher ${ }^{5}$ for a general class of pressure-controlled valves. Yoshikawa ${ }^{6}$ measured vibration patterns of horn players and observed the switch between outward-striking and sideways-striking modes in relation to the blowing pitch. This observation was also confirmed by Copley and Strong ${ }^{7}$ and Chen and Weinreich. ${ }^{8}$ Results of computer simulations for the trumpet were presented by Adachi and Sato ${ }^{9,10}$ using one- and two-dimensional motion models. In addition, their oscillation scheme was debated using regeneration theory. ${ }^{11}$ Other fac-

\footnotetext{
a) Author to whom correspondence should be addressed. Electronic mail: kabu@design.kyushu-u.ac.jp
}

tors of the player's performance were investigated by Fletcher and Tarnopolsky ${ }^{12}$ in terms of the blowing pressure, which depends on the blowing pitch and affects the loudness and timbre.

The player's vocal tract constitutes an acoustic resonator connected to the instrument via the reed generator. At frequencies near resonances of the instrument bore, the input impedance of the vocal tract is usually smaller in magnitude than the impedance of the bore. Therefore in many situations, the acoustic effect of the vocal tract can to a first approximation be ignored, and it has no explicit effect on the vibration of lips and tone of the instrument. This prediction was experimentally supported by Backus ${ }^{13}$ for woodwind instruments. However, Scavone et al. ${ }^{14}$ and Chen et al. ${ }^{15}$ noted the influence of the vocal tract in pitch bending of the saxophone and clarinet. In this specific performance, the magnitude of the measured impedance of the vocal tract was comparable to that of the bore resonance. On the basis of these findings, they concluded that the resonance characteristics of the vocal tract can influence, select, or control the frequency of reed vibrations.

In the case of brass instruments, on the other hand, it is frequently said that skilled players empirically change the shape of the vocal tract to control the pitch, timbre, and articulation, ${ }^{16}$ suggesting possible acoustic roles of the vocal 
tract as found for woodwind instruments. ${ }^{17}$ Especially in the high register, there is the possibility that the vocal tract can create a resonance strong enough to influence the vibratory motion of the lips because the peaks in the input impedance of the trumpet becomes small in the high frequency region. ${ }^{1}$

A thorough method is presented in this paper to investigate the problem quantitatively. In addition, preliminary results of morphological and acoustic investigations are shown using a professional trumpet player to demonstrate the effectiveness of the method and consider the possible acoustic role of the player's vocal tract. The shape of the vocal tract is generally difficult to observe non-invasively. Therefore magnetic resonance imaging (MRI) is used to provide a high-resolution volumetric image of the entire vocal tract while playing. As well as the morphological information, the area function is estimated from volumetric data, and the input impedance is computed using an acoustic model of the lossy vocal tract. ${ }^{18}$ Finally, a time-domain numerical simulation of a blowing trumpet is performed using a model of the lips. ${ }^{10}$ Because the blowing condition can be changed flexibly in the computer simulation, we examine the influence of the vocal tract on the sound pitch and blowing pressure in a systematic manner and show how the control of the vocal-tract shape can be effective in playing a trumpet. In this study, the definition of the player's air column includes the subglottal tract as well as the supraglottal tract (vocal tract) because they are coupled together via the opened glottis.

\section{MORPHOLOGICAL OBSERVATION OF THE VOCAL TRACT}

Observation of the vocal tract is important to investigate its configuration during an actual performance. A number of techniques are applicable for observing the shape or movement of the vocal tract, such as x-ray, ultrasonic pulsedecho, magnetic motion capturing, and MRI techniques. ${ }^{19}$ MRI is capable of non-invasively imaging the vocal-tract configuration in three dimensions, and it has already been used in estimating the area function and acoustic resonance characteristics in the production of speech. ${ }^{20}$ Therefore a measurement of the vocal tract was conducted using MRI for actual performance of the trumpet. Sectional images of the vocal tract were scanned for a professional trumpet player in three dimensions as the player blew into an instrument for a number of musical pitches. The subject was a Japanese male having a professional career as a trumpet player of more than $30 \mathrm{yr}$.

Because the MRI system produces a strong magnetic field, metallic materials are unusable. An instrument was therefore fabricated using polyvinyl chloride tubes with the help of information about the bore shape of an actual $\mathrm{B}^{\mathrm{b}}$ trumpet (Yamaha YTR-2320E, student model), where the valves were supposed to be open. ${ }^{9}$ Although the axial length was correctly designed for the fabricated instrument, the sectional area was approximated by concatenating cylindrical tubes of uniform sectional area. For the opening of the instrument (bell), tubes like a truncated cone were used to mimic the abrupt increase in area. The mouthpiece was made of acrylic.

In addition, the shape of the upper and lower teeth was measured independently because they were as transparent as air in the standard vocal-tract scanning. Following the method of Takemoto et al., ${ }^{21}$ a contrast medium was inserted in the oral cavity of the player so that the outline of the teeth was distinguished from the oral cavity. The oral shape was then scanned for the rest state of the vocal tract without blowing according to usual scanning procedure.

The measurement condition was as follows. Each slice of the volumetric data was taken on a sagittal plane of the subject. The slice thickness was $2 \mathrm{~mm}$ for the vocal tract during blowing and $1.5 \mathrm{~mm}$ for observing the teeth shape. The numbers of slices were 30 for the vocal tract and 51 for the teeth. Because it required a scanning time of about 30-40 s to obtain a set of volumetric data, the player maintained the configuration of the vocal tract as stably as possible during the scanning. The resolution of each slice was $512 \times 512$ pixels covering a scanning area of $256 \times 256 \mathrm{~mm}$. The measurement and ethical judgment were made by the ATR Brain Activity Imaging Center (Kyoto, Japan).

Figure 1 shows the measured midsagittal images of the player's vocal tract for three pitches corresponding, from left to right, to $\mathrm{E}_{4}^{b}, \mathrm{~B}_{4}^{b}$, and $\mathrm{F}_{5}$. The blowing frequency was 311 , 466, and $698 \mathrm{~Hz}$, respectively. Main speech articulators,
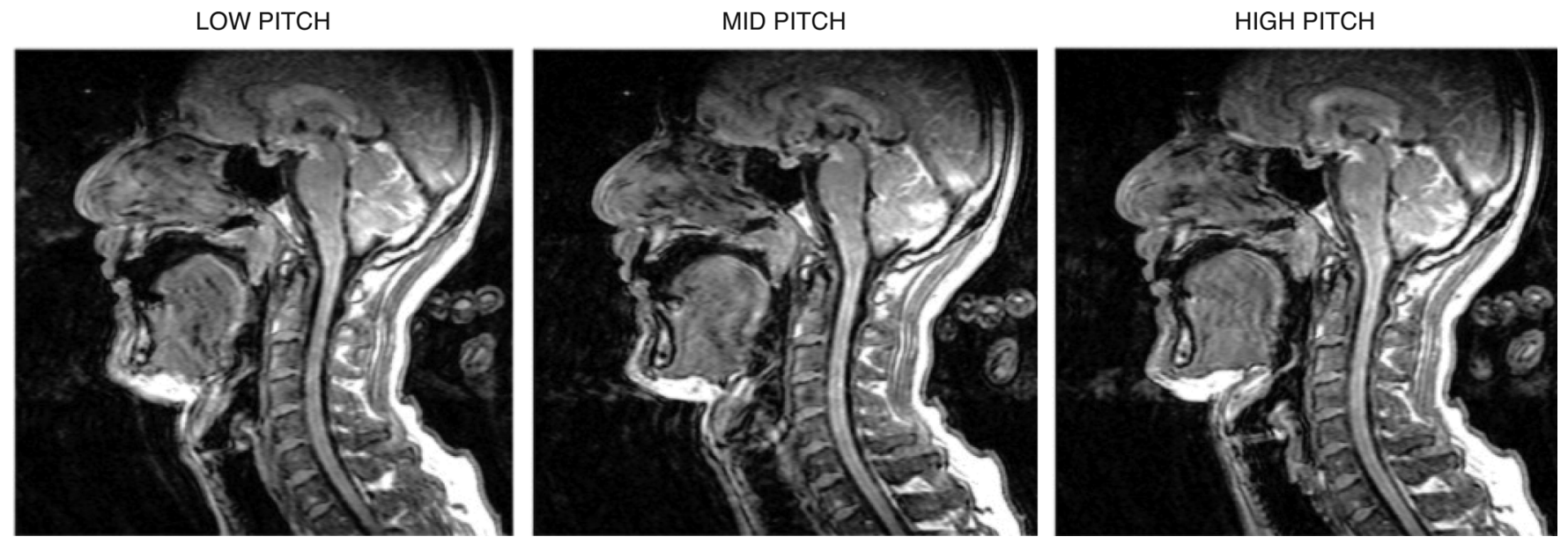

FIG. 1. Midsagittal image of the vocal tract of a professional trumpet player corresponding, from left to right, to low $\left(\mathrm{E}_{4}^{b}\right)$, mid $\left(\mathrm{B}_{4}^{b}\right)$, and high $\left(\mathrm{F}_{5}\right)$ pitches. 
lo/

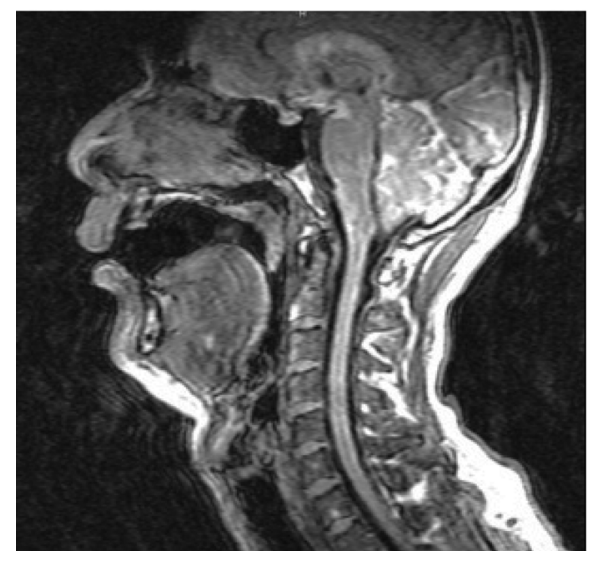

lul

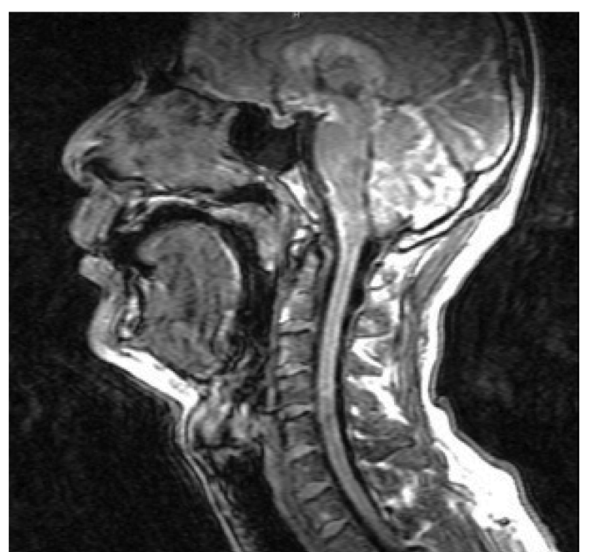

le/

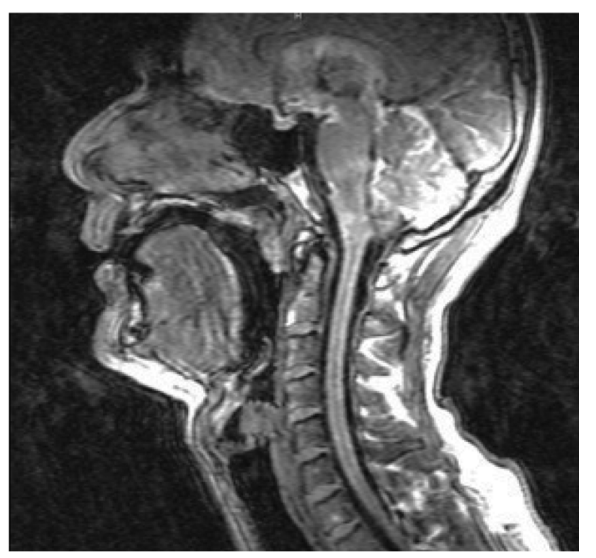

FIG. 2. Midsagittal image of the vocal tract during the production of the Japanese vowels /o/ (left), /u/ (center), and /e/ (right).

such as the tongue, lips, and soft palate, are observed clearly in the images. For the low and mid pitches, the tongue was located slightly back in the tract, making a dominant vocaltract constriction between the tongue and soft palate. When the pitch was high, the tongue was positioned more anteriorly, and the vocal tract was constricted near the border of the hard and soft palates. To compare the posture of the vocal organs in Fig. 1 with those during the production of speech, images are shown in Fig. 2 for the sustained Japanese vowels $/ \mathrm{o} /, / \mathrm{u} /$, and $/ \mathrm{e} /$ recorded for the same subject. The figures show that the tongue posture for the low and mid pitches was similar to that for the back vowel /o/, but it was at a slightly anterior position. As a result, the cavity in front of the constriction was smaller. The tongue posture for the high-pitch trumpet sound was similar to that for the vowel $/ \mathrm{u} /$, but it was located slightly posteriorly.

The low pitch produced by the player, $\mathrm{E}_{4}^{b}$, was not standard for a $\mathrm{B}^{b}$ trumpet with valves open. This discrepancy was possibly due to the incompleteness of the fabricated instrument; e.g., there was error in the cross dimension and different materials were used. For example, the diameter of the bell opening was about $10.5 \mathrm{~cm}$ and smaller than the actual instrument $(12.37 \mathrm{~cm}$ for the Yamaha YTR-2320E). However, through a numerical calculation of the input impedance, we confirmed that the error in the cross dimension of the fabricated instrument would not yield such an obvious pitch error if the wall was assumed to be rigid. The softness of the fabricated instrument and the loss factor due to the wall motion were therefore guessed as the main cause of the pitch error.

Next, the area function of the vocal tract was estimated using the volumetric images. The volumetric data for the upper and lower teeth were first superimposed on the vocaltract data following the method of Takemoto et al. ${ }^{21}$ The superposition was based on an affine congruent transformation. The transformation matrix was determined from four anatomical landmarks visible in the teeth and tract images, such as the nasal spine and surface of a molar tooth. However, positioning of these landmarks by sight suffers from artificial errors. To refine the transformation matrix, the optimal landmark positions were searched for using a computer in the vicinity of the initial points, instead of employing simulated annealing. ${ }^{21}$ As the error criterion of this search procedure, we used an a priori idea that the teeth portion is dark. Before applying the affine transformation, the teeth portion was therefore marked manually in the images. When the transformation was performed and the marked teeth portion was mapped onto the tract images, the mean pixel value corresponding to the teeth portion was calculated. The optimal landmark positions, and hence the optimal affine transformation, were determined so that this mean pixel value was smallest.

The area function was then obtained using the vocaltract images with the teeth superimposed. First, the inferior and superior outlines of the vocal tract were traced by sight on the midsagittal plane. The center line of the tract, representing the axis of wave propagation, was determined so that the distances of each node point on the center line from the two outlines were equal. The vocal tract was then divided along the center line into 40 sections of equal length. Finally, the cross sectional area in the direction perpendicular to the center line was obtained for each section.

The area-function data are plotted in Fig. 3. The figure shows that as the pitch changed from low to mid-level and from mid-level to high, the sectional area decreased for
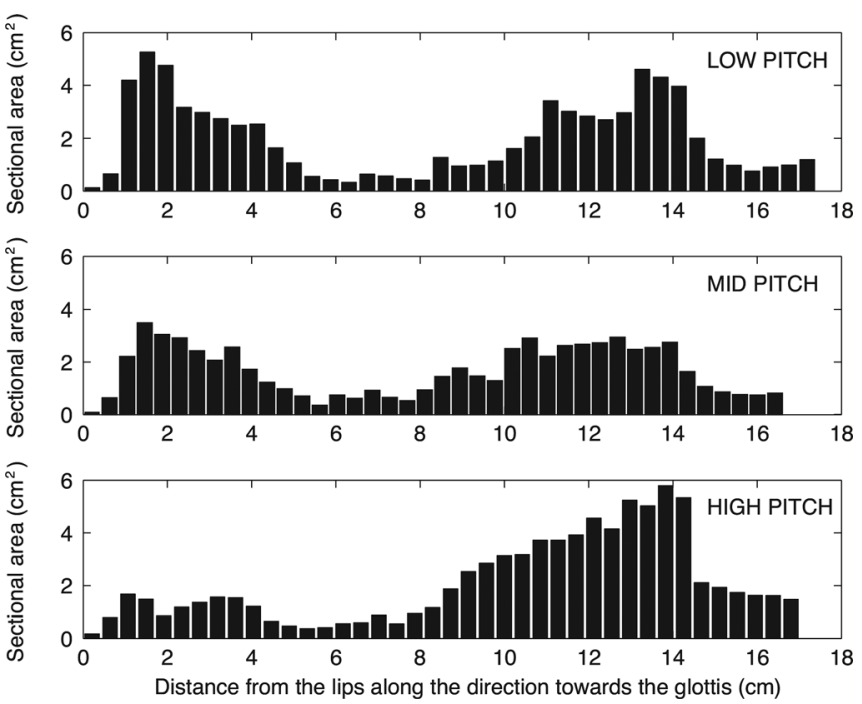

FIG. 3. Vocal-tract area function obtained from volumetric MRI. From top to bottom, the plots correspond to low, mid, and high pitches. 
sections anterior to the vocal-tract constriction made between the tongue and the soft palate. This volume reduction near the gum and the hard palate was the result of the raising of the tongue observed in Fig. 1. To the contrary, the area of sections posterior to this constriction increased noticeably when the pitch was high. The length of the vocal tract was estimated as $17.4 \mathrm{~cm}$ for the low, $16.6 \mathrm{~cm}$ for the mid, and $17.0 \mathrm{~cm}$ for the high pitches. The larynx rose slightly for the mid pitch, decreasing the vocal-tract length. The area and length of the glottis were estimated as 0.14 $\mathrm{cm}^{2}$ and $0.12 \mathrm{~cm}$, respectively, from MRI of the larynx.

\section{IMPEDANCE OF THE PLAYER'S AIR COLUMN}

\section{A. Impedance calculation}

Next, the acoustic property of the player's air column is estimated using the area-function data in terms of the input impedance seen from the lips. By assuming plane wave propagation, the input impedance can be calculated as follows using an acoustic tube model, where the column is represented as the concatenation of multiple cylinders:

$$
Z_{\mathrm{vt}}=-P_{\mathrm{a}} / U_{\mathrm{a}}
$$

where $P_{\mathrm{a}}$ and $U_{\mathrm{a}}$ are the Fourier transforms of the acoustic pressure and volume velocity. For waves propagating from the mouth to the instrument, the direction is taken as positive. Using a frequency-domain model of the acoustic tube including wall losses, ${ }^{18}$ the input impedance is expressed as

$$
Z_{\mathrm{vt}}=-\frac{A Z_{\mathrm{p}}+B}{C Z_{\mathrm{p}}+D}
$$

where the matrix chain is given as

$$
\left(\begin{array}{ll}
A & B \\
C & D
\end{array}\right)=\left(\begin{array}{ll}
A_{1} & B_{1} \\
C_{1} & D_{1}
\end{array}\right)\left(\begin{array}{ll}
A_{2} & B_{2} \\
C_{2} & D_{2}
\end{array}\right) \cdots\left(\begin{array}{cc}
A_{N} & B_{N} \\
C_{N} & D_{N}
\end{array}\right) .
$$

The entire air column including the sub- and supraglottal tracts is modeled here because the glottis is constantly open during the blowing, and the vocal tract can acoustically be coupled with the subglottal system. The entire column was represented by the cascade connection of cylindrical tubes. In the matrix chain given in Eq. (3), the first tube section corresponds to the lips and the $N$ th to the lungs. Components $A_{i}$, $B_{i}, C_{i}$, and $D_{i}$ for the $i$ th section are determined by the length, $L_{i}$, and sectional area, $S_{i}$, using the model of Sondhi and Schroeter, ${ }^{18}$ such that $A_{i}=\cosh \left(\sigma L_{i} / c\right), \quad B_{i}=-(\rho c /$ $\left.S_{i}\right) \gamma \sinh \left(\sigma L_{i} / c\right), \quad C_{i}=-\left(S_{i} / \rho c\right)\left(\sinh \left(\sigma L_{i} / c\right)\right) / \gamma$, and $D_{i}$ $=\cosh \left(\sigma L_{i} / c\right) . \alpha, \beta, \gamma$, and $\sigma$ are frequency-dependent parameters given in the literature, such as $\alpha=\sqrt{j \omega c_{1}}, \beta=j \omega \omega_{0}^{2} /$ $\{(j \omega+a) j \omega+b\}+\alpha, \quad \gamma=\sqrt{(\alpha+j \omega) /(\beta+j \omega)}, \quad$ and $\sigma=\gamma(\beta+j \omega)$, where $a=130 \pi \mathrm{rad} / \mathrm{s}, b=(30 \pi)^{2}(\mathrm{rad} / \mathrm{s})^{2}$, $c_{1}=4 \mathrm{rad} / \mathrm{s}, \omega_{0}^{2}=(406 \pi)^{2}(\mathrm{rad} / \mathrm{s})^{2}$, and $j=\sqrt{-1} . \rho$ is the air density and $c$ is the speed of sound. $Z_{\mathrm{p}}=\rho c^{2} /(j \omega V)$ in Eq. (2) is the terminal impedance modeled as a capacity corresponding to an air volume, $V$, of $500 \mathrm{ml}^{24}$

The area-function data for the vocal tract were given in the previous section (Fig. 3). The glottis was represented by a uniform tube with an area of $0.14 \mathrm{~cm}^{2}$ and length of $0.12 \mathrm{~cm}$. For the subglottal tract, the morphological data were obtained by Weibel. ${ }^{23}$ The subglottal tract is classified into conductive, transitory, and respiratory zones, where the sectional area is relatively constant along the section of about $20 \mathrm{~cm}$ below the glottis but increases rapidly in the remainder of the conductive zone and in the transitory and respiratory zones. The branching of airways was not exactly considered in this study, and the cross-sectional area in each stage was summed to form single uniform tube as shown in Fig. 6 of Ishizaka et al. ${ }^{22}$ The resulting area-function data were then used to calculate the player's impedance, where the total length of the subglottal tract was shortened uniformly by a factor of $0.941 .^{22}$ In addition, the wall loss is greater for the subglottal tract than that for the vocal tract because of an effective increase in the wall area. This effect was approximately taken into consideration by changing the parameter value of the acoustic tube model as $a=780 \pi \mathrm{rad} / \mathrm{s}$ and $c_{1}=16 \mathrm{rad} / \mathrm{s}$ so that the input impedance of the subglottal tract seen from the glottis agrees with the numerical data presented in the literature. ${ }^{22}$

The numerical results are shown in Fig. 4, where the top plots present the magnitude and the bottom plots the phase angle. The vertical, broken line indicates the blowing frequency for each pitch. The magnitude data show that the input impedance has three peaks below $1 \mathrm{kHz}$. In each pitch condition, the first and third peaks were originated from the resonances of the vocal tract while the second one from that of the subglottal tract. The lowest peak has maximum amplitude for the low and mid pitches, and the third one is the maximum for the high pitch. The blowing pitch for the low note agreed well with the largest peak in the impedance, and we see that the frequency of the largest peak for the high note increased significantly. Titze ${ }^{25}$ examined the effect of coupling between the voice generation system and the vocal tract and showed that the subglottal tract having a compliant acoustic load is favorable for vocal fold oscillation. The player's air column may have a similar effect on the performance of the trumpet, but, for this particular player, the phase is obviously positive for the low and high pitches at each blowing frequency. This problem is discussed again in the following text in terms of the total impedance.

\section{B. Total impedance including player and instrument}

The oscillation condition for woodwind and brass instruments has been investigated in terms of regeneration theory. ${ }^{11,26}$ By assuming a linearized reed-instrument model, the theory implies a constraint that the frequency responses of the reed and instrument should satisfy. Adachi and Sato stated the constraint as $\sqrt{2 p_{0} / \rho}|G(f)||Z(f)| \geq 1$ and $\angle G(f)+\angle Z(f)=0$ in Eqs. (12) and (13) of the literature, ${ }^{10}$ where $p_{0}$ is the aerodynamic pressure supplied by the lungs. $G(f)$ is the lip mobility representing the response of the lips to the driving pressure as a function of the frequency, $f . Z(f)$ is the input impedance of the trumpet in Adachi and Sato, ${ }^{10}$ but in the original study regarding regeneration theory, ${ }^{11} Z(f)$ represents the total impedance including the trumpet and player's air column.

The input impedance of the trumpet, $Z_{\mathrm{trp}}$, was numerically estimated and the magnitude of the total impedance, $Z_{\mathrm{trp}}+Z_{\mathrm{vt}}$, 

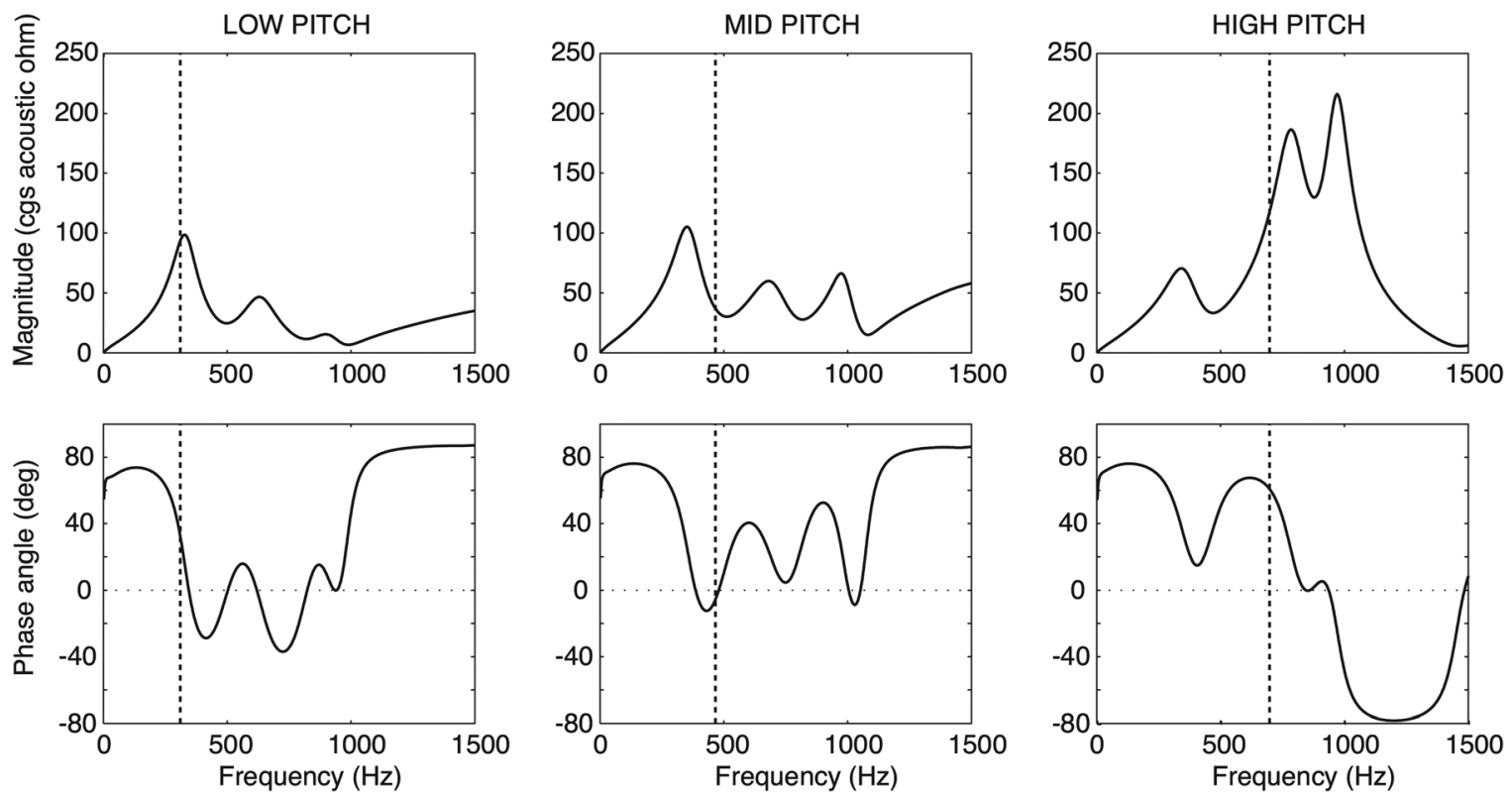

FIG. 4. Calculated input impedance (magnitude and phase) of the player's air column, $Z_{\mathrm{vt}}$, including the vocal tract, glottis, and sub-glottal tract, seen from the lips. From left to right, the plots correspond to low, mid, and high pitches. The vertical broken line indicates the blowing frequency for each pitch.

is shown in Figs. 5(B) and 5(C) by adding the player's impedance, $Z_{\mathrm{vt}}$, plotted in Fig. 4 . The magnitude of $Z_{\text {trp }}$ is also shown by the broken line. The air column of a mouthpiece cup and a $\mathrm{B}^{b}$ trumpet (Yamaha YTR-2320E, student model) with the valves open [Fig. 5(A)] was modeled here by concatenating truncated cones. ${ }^{27}$ Adachi and Sato $^{9}$ showed that the numerical result agrees well with the data obtained by acoustic measurements. ${ }^{1}$

The total impedance was basically determined by the trumpet itself and unaffected by the player's impedance. The peaks located at 234, 345, 463, 580, 695, 811, and $924 \mathrm{~Hz}$ are resonances of the instrument with mode numbers from two to eight, respectively. The first resonance is omitted because it is not used in standard trumpet performance. The blowing frequency of the player in the MRI experiment was $466 \mathrm{~Hz}$ for the mid pitch and $698 \mathrm{~Hz}$ for the high pitch. The figure shows that these frequencies were consistent with the fourth and sixth resonance frequencies. On the other hand, the blowing frequency for the low pitch $(311 \mathrm{~Hz})$ was lower than the third resonance frequency corresponding to $\mathrm{F}_{4}$. As explained before, this discrepancy was possibly due to the incompleteness of the instrument fabricated for the MRI experiment. Only for the high-pitch vocal-tract condition, the peaks of the player's impedance around 800 and $1000 \mathrm{~Hz}$ caused a slight increase in the total impedance for the range greater than $750 \mathrm{~Hz}$. From the condition $\sqrt{2 p_{0} / \rho}|G(f)||Z(f)| \geq 1$, this increase in magnitude may assist the oscillation of the lips.

The phase property of the total impedance is shown in Fig. 6. For each resonance, the phase angle changes from positive to negative. The figure shows that the change in phase is more apparent than that in magnitude. Due to the incorporation of the player's impedance, the range of the phase angle changing from positive to negative becomes slightly narrower, implying that the real, resistive component increased relative to the reactance by considering that both components are connected in a series. The effect was dominant between the second and fourth resonances for the low and mid pitches, and under the high-pitch vocal-tract condition, the figure shows that the effect spread to higher resonances.

\section{INFLUENCE OF THE VOCAL TRACT UPON LIP MOTION}

Finally, a total sound production model of the trumpet is presented to investigate the effect of the vocal tract on the vibration of the lips. The model comprises a two-dimensional model of the lips, ${ }^{10}$ equations for flow in the vicinity of the lips, and the acoustic characteristics of the instrument $\left(Z_{\text {trp }}\right)$ and the player's air column $\left(Z_{\mathrm{vt}}\right)$ including the vocal tract, glottis, and subglottal tract. The lip motion changes the sectional area at the lip nozzle and controls the volume flow as a dynamic valve. Inversely, the flow passing through the nozzle provides the driving force to the lips and induces their self-oscillation. The acoustic load of the instrument also affects the oscillation of the lips strongly. We also consider the source-filter interaction, ${ }^{25}$ i.e., the feedback effect of the acoustic pressure from the mouth on the lip motion.

\section{A. Simulation method}

The blowing simulation is based on the model of Adachi and Sato ${ }^{10}$ employing two-dimensional motion of the lips (Fig. 7), which can be regarded as the combination of the outwardstriking and sideways-striking modes. ${ }^{5}$ The outward-striking mode is mainly driven by the pressure difference between the mouth and the mouthpiece cup, $p_{\mathrm{vt}}-p_{\text {cup }}$, while the sidewaysstriking mode is mainly driven by the pressure in the lip nozzle, $p_{\text {lip. }}$. The model assumes symmetrical motion of both lips, and the equation of motion with respect to the displacement of a mass placed at the center of the lips is given as ${ }^{10}$

$$
\frac{1}{2} m \frac{d^{2} \vec{\xi}}{d t^{2}}=-\frac{1}{2} \frac{\sqrt{m k}}{Q} \frac{d \vec{\xi}}{d t}+\vec{F}_{\text {restore }}+\vec{F}_{\Delta \mathrm{p}}+\vec{F}_{\text {Bernoulli }}
$$


(A)
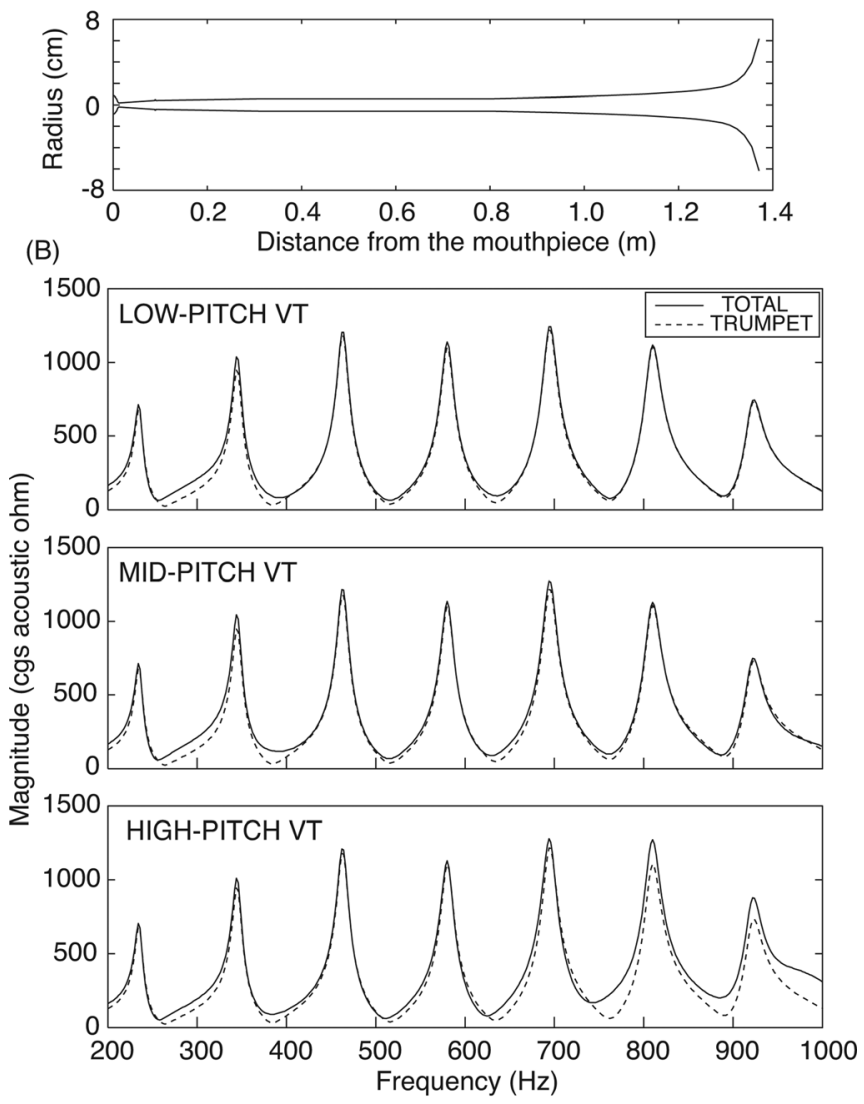

(C)

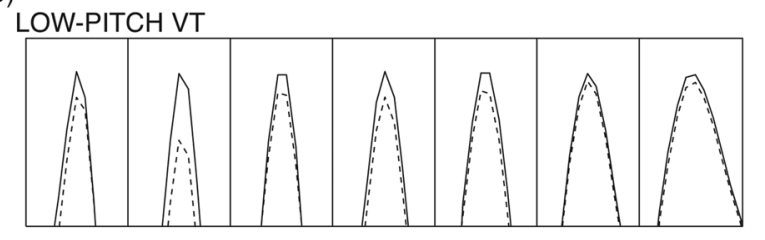

MID-PITCH VT
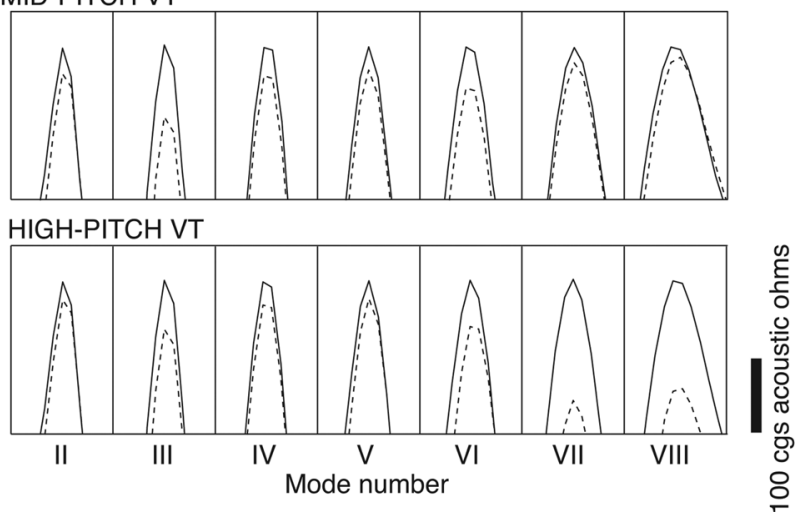

FIG. 5. The interior radius of the mouthpiece cup and a $\mathrm{B}^{\mathrm{b}}$ trumpet is plotted in (A) as a function of the distance from the mouthpiece. The magnitude of the total impedance, $Z_{\mathrm{trp}}+Z_{\mathrm{vt}}$, including the trumpet and the player's air column is plotted in (B) with the solid line for three vocal tract shapes corresponding to low, mid, and high pitches. The broken lines indicate the magnitude of $Z_{\text {trp }}$. VT is an abbreviation of vocal tract. The plot in (C) shows the close-up of peaks, corresponding to modes from two to eight, in the total (solid lines) and instrument (broken lines) impedances with a magnifying factor of five, both in the horizontal and vertical directions.

where $\vec{\xi}$ is the displacement measured at point $\mathrm{C}, m$ is the mass, $k$ is the stiffness, and $Q$ is the quality value. The restoring force, $\vec{F}_{\text {restore, }}$ and external forces arising from the pressure difference and the Bernoulli force, $\vec{F}_{\Delta \mathrm{p}}$ and $\vec{F}_{\text {Bernoulli, are, }}$ respectively, given by Eqs. (2) through (4) of the literature ${ }^{10}$ as $\quad \vec{F}_{\text {restore }}=-0.5 k\left(\vec{\xi}-\vec{\xi}_{\text {equil }}\right), \quad \vec{F}_{\Delta \mathrm{p}}=b\left(p_{\mathrm{vt}}-p_{\text {cup }}\right)(\vec{\xi}$ $\left.-\vec{\xi}_{\text {joint }}\right)^{\perp}$, and $\vec{F}_{\text {Bernoulli }}=b d p_{\text {lip }} \vec{e}_{y}$, where $p_{\mathrm{vt}}$ is the mouth pressure, $p_{\text {cup }}$ is the mouthpiece cup pressure, $p_{\text {lip }}$ is the lip nozzle pressure, $b$ and $d$ are length and thickness of the lips, $\vec{\xi}_{\text {equil }}$ is the rest position, $\vec{\xi}_{\text {joint }}$ is the position of point $\mathrm{B}, \vec{e}_{y}$ is the unit vector along the $y$ axis, and $\perp$ represents the orthogonal component. $Q$ is set to 3.0 when the lips are open and 0.5 when they are closed. As a distinctive feature, we consider wave propagation in the player's air column in addition to the trumpet bore. Therefore, the mouth pressure, $p_{\mathrm{vt}}$, is given as

$$
p_{\mathrm{vt}}=p_{0}+p_{\mathrm{a}}
$$

where $p_{0}$ is the aerodynamic pressure supplied by the lungs and $p_{\mathrm{a}}$ is the acoustic pressure immediately upstream of the lips.

We next consider the estimation of the volume flow rate, $U$, that passes through the lip nozzle. Following the Adachi-Sato model, $U$ is given as $U=U_{\mathrm{a}}+U_{\text {lip }}$, where $U_{\mathrm{a}}$ is the flow component generated by the pressure difference between points upstream and downstream of the lips. $U_{\text {lip }}$ is the flow swept by the movement of the lips calculated as a function of the lip displacement [Eq. (6) of the literature]. Therefore if the constant lung pressure, $p_{0}$, is specified as a simulation condition, and if the lip displacement, $\vec{\xi}$, is solved using the equation of motion, the values of $p_{\mathrm{a}}, p_{\text {lip }}, p_{\text {cup }}$, and $U_{\mathrm{a}}$ are not known. Assuming that the flow is inviscid upstream of the boundary between the lips and the mouthpiece cup [region (a) shown in Fig. 7] and applying the momentum conservation law downstream of this boundary [region (b)], the quantities are determined by solving the following equations simultaneously:

$$
\begin{aligned}
& p_{\mathrm{vt}}-p_{\text {lip }}=\frac{1}{2} \rho\left(\frac{U_{\mathrm{a}}}{S_{\text {lip }}}\right)^{2}+\frac{\rho d}{S_{\text {lip }}} \frac{\mathrm{d} U_{a}}{\mathrm{dt}}, \\
& p_{\text {lip }}-p_{\text {cup }}=-\rho U_{\mathrm{a}}^{2}\left(\frac{1}{S_{\text {cup }} S_{\text {lip }}}-\frac{1}{S_{\text {cup }}^{2}}\right), \\
& p_{\text {cup }}=z_{\text {trp }} * U,
\end{aligned}
$$

and

$$
p_{\mathrm{a}}=z_{\mathrm{vt}} *(-U),
$$

where $S_{\text {lip }}=\max \left(2 b \vec{\xi}^{\perp}, 0\right)$ is the sectional area of the lip nozzle and $S_{\text {cup }}$ is the area of the entrance of the mouthpiece. $z_{\mathrm{vt}}$ and $z_{\text {trp }}$ are the inverse Fourier transforms of the input impedances for the player and instrument. The operator $*$ denotes the convolution. Equations (6) and (7), respectively, correspond to Eqs. (7) and (8) of the literature, ${ }^{10}$ except that the mouth pressure, $p_{\mathrm{vt}}$, includes the acoustic component, $p_{\mathrm{a}}$. This acoustic component is related to the volume flow via the player's input impedance in Eq. (9).

\section{B. Results}

The blowing of the trumpet was simulated as follows. Given the blowing pressure $p_{0}$, the values of the physical 

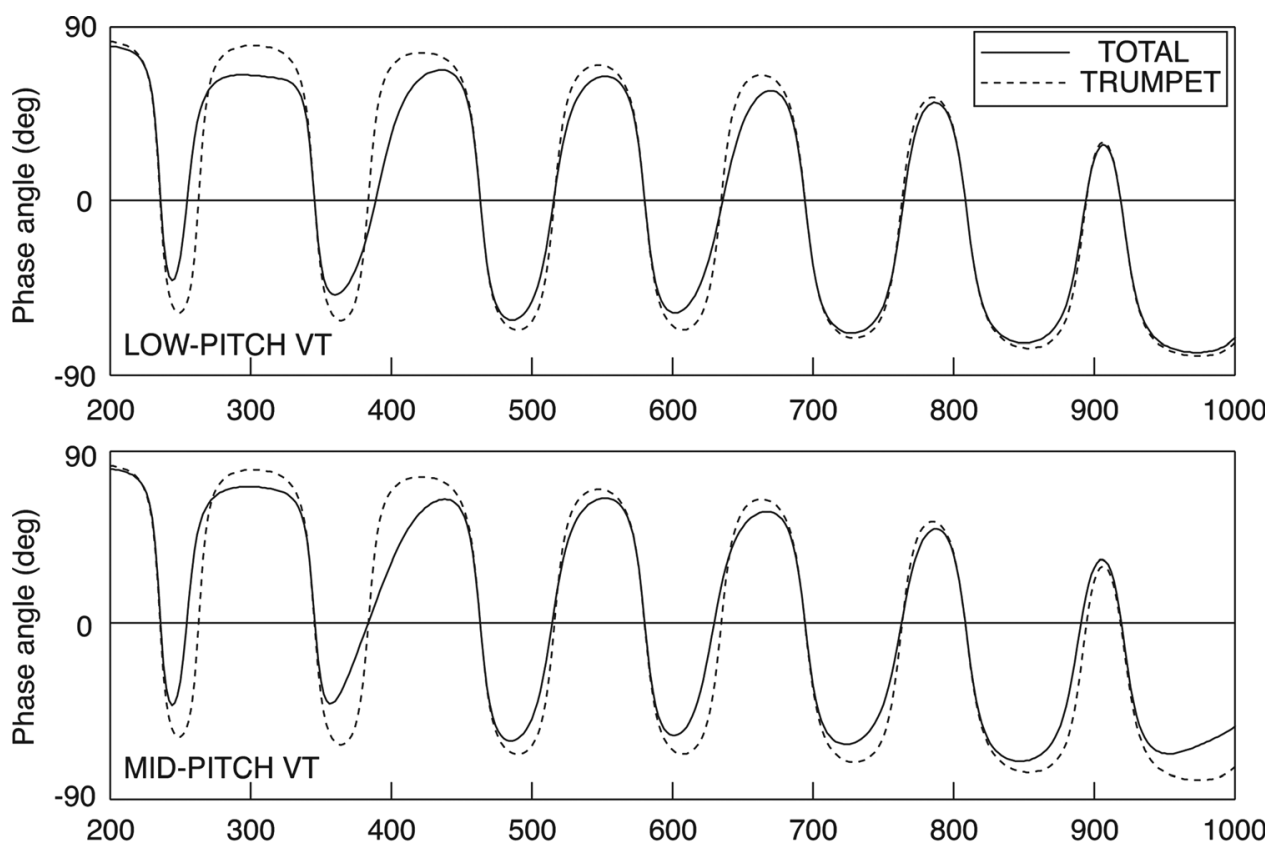

FIG. 6. The phase of the total impedance, $Z_{\text {trp }}+Z_{\mathrm{vt}}$, is plotted with the solid line for three vocal tract shapes corresponding to low, mid, and high pitches. The broken lines indicate the phase of $Z_{\text {trp }}$.

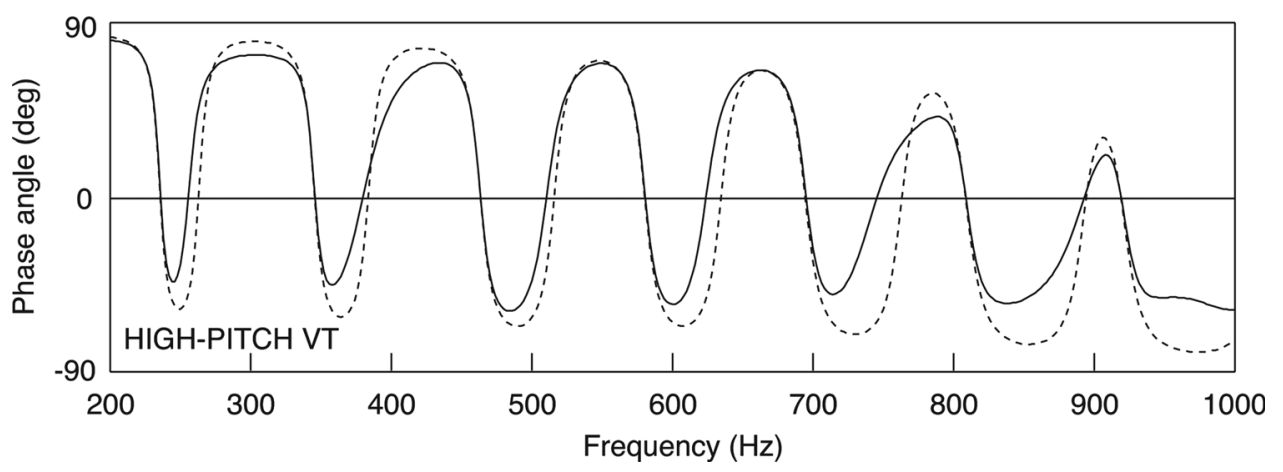

variables $\vec{\xi}, p_{\mathrm{a}}, p_{\text {lip }}, p_{\text {cup }}, U_{\mathrm{a}}$, and $U_{\text {lip }}$ were initially set to zero. $z_{\mathrm{vt}}$ and $z_{\mathrm{trp}}$ were obtained from the area function of the player's air column and the instrument bore. The restoring and external forces in Eq. (4) were then calculated, and the equation of the lip motion was solved numerically employing a finite difference method. From the new value of $\vec{\xi}, U_{\text {lip }}$, and

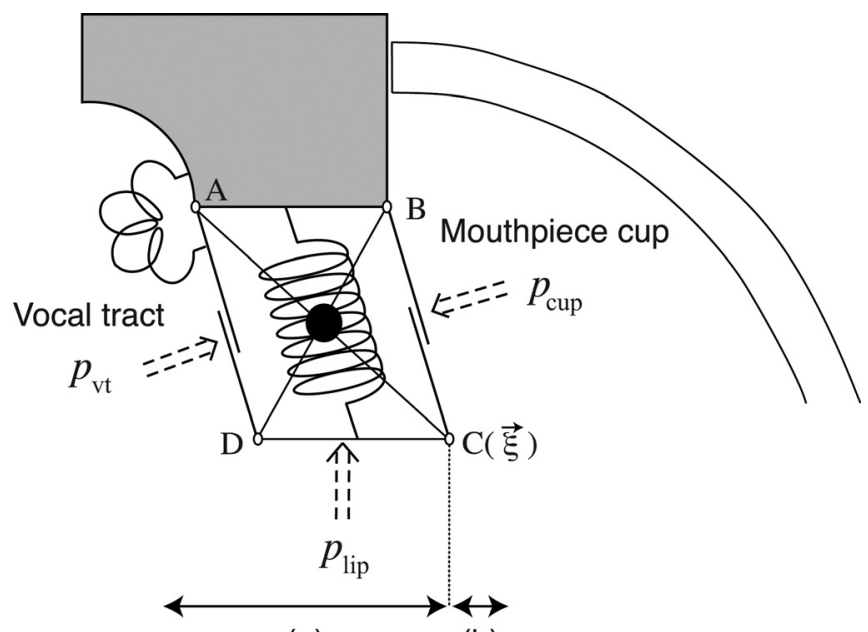

(a)

(b)

FIG. 7. Two-dimensional mechanical model of the lips (Ref. 10). The mass is located at the center of the parallelogram $\mathrm{ABCD}$. Therefore the displacement of the mass is half that for the point $\mathrm{C}$ indicated as $\vec{\xi}$.
$S_{\text {lip }}$ were calculated. Next the values of the aerodynamic and acoustic variables, $p_{\mathrm{a}}, p_{\text {lip }}, p_{\text {cup }}$, and $U_{\mathrm{a}}$, were determined using the relation given in Eqs. (6) through (9). Finally, the total volume flow rate, $U$, was obtained as the sum of $U_{\mathrm{a}}$ and $U_{\text {lip }}$. The procedure was repeated for the desired time steps. The values of the model parameters were set in accordance with Table I of the original paper ${ }^{10}$ such that $c=340 \mathrm{~m} / \mathrm{s}$, $\rho=1.2 \mathrm{~kg} / \mathrm{m}^{3}, S_{\text {cup }}=2.3 \times 10^{-4} \mathrm{~m}^{2}, b=7.0 \times 10^{-3} \mathrm{~m}$, $d=2.0 \times 10^{-3} \mathrm{~m}, \quad \vec{\xi}_{\text {joint }}=(0,4.0) \times 10^{-3} \mathrm{~m}$, and $\vec{\xi}_{\text {equil }}$ $=(1.0,-2.1) \times 10^{-3} \mathrm{~m}$. The lip eigenfrequency, $f_{\text {lip }}$, was varied extensively from 220 to $1000 \mathrm{~Hz}$ with a step size of 20 Hz. From the relation $f_{\text {lip }}=\sqrt{k / m} / 2 \pi$, the stiffness, $k$, was set in proportion to the eigenfrequency $\left(k=1.5 f_{\text {lip }} \mathrm{N} / \mathrm{m}\right)$ and the mass, $m$, inversely proportional to the eigenfrequency $\left[m=1.5 /\left\{(2 \pi)^{2} f_{\text {lip }}\right\} \mathrm{kg}\right]$. In addition, the standard blowing pressure was set, as summarized in Table I, for each value of the lip eigenfrequency. The sampling frequency was $64 \mathrm{kHz}$.

To examine how the vocal tract can affect the vibration of the lips, the condition of the feedback acoustic pressure, $p_{\mathrm{a}}$, was changed as follows. First, as the base condition, $p_{\mathrm{a}}$ was constantly set to zero, meaning there was no acoustic feedback from the player's vocal tract. The effect of the vocal tract was taken into consideration under other conditions. The shape of the vocal tract was given by the area function for the low, mid, or high pitches (Fig. 3). The input impedance, $Z_{\mathrm{v} t}$, was then calculated (Fig. 4) and the 
TABLE I. Blowing pressure settings. $f_{\text {lip }}$ is the lip eigenfrequency and $p_{0}$ is the corresponding blowing pressure from the lungs.

\begin{tabular}{lc}
\hline \hline$f_{\text {lip }}(\mathrm{Hz})$ & $p_{0}(\mathrm{kPa})$ \\
\hline $220-260$ & 3.0 \\
$280-380$ & 4.5 \\
$400-520$ & 5.5 \\
$540-640$ & 7.0 \\
$660-780$ & 8.5 \\
$800-920$ & 11.5 \\
$940-960$ & 12.0 \\
980 & 14.0 \\
1000 & 16.0 \\
\hline \hline
\end{tabular}

feedback pressure, $p_{\mathrm{a}}$, was estimated using the relation given in Eq. (9). The simulation results are shown in Fig. 8, where the vibration frequency of the lips is plotted as a function of the eigenfrequency.

For the base condition shown at upper left in the figure, the simulated vibration frequency is indicated by circles. The vibration frequency has a stepwise pattern for the change in eigenfrequency, but in agreement with the original work, ${ }^{10}$ it is not so different from the eigenfrequency and very close to one of the mode frequencies shown by broken, horizontal lines with the mode numbers. For the seventh and eighth modes, the vibration frequency is lower than the eigenfrequency. This is a typical feature of the transversal
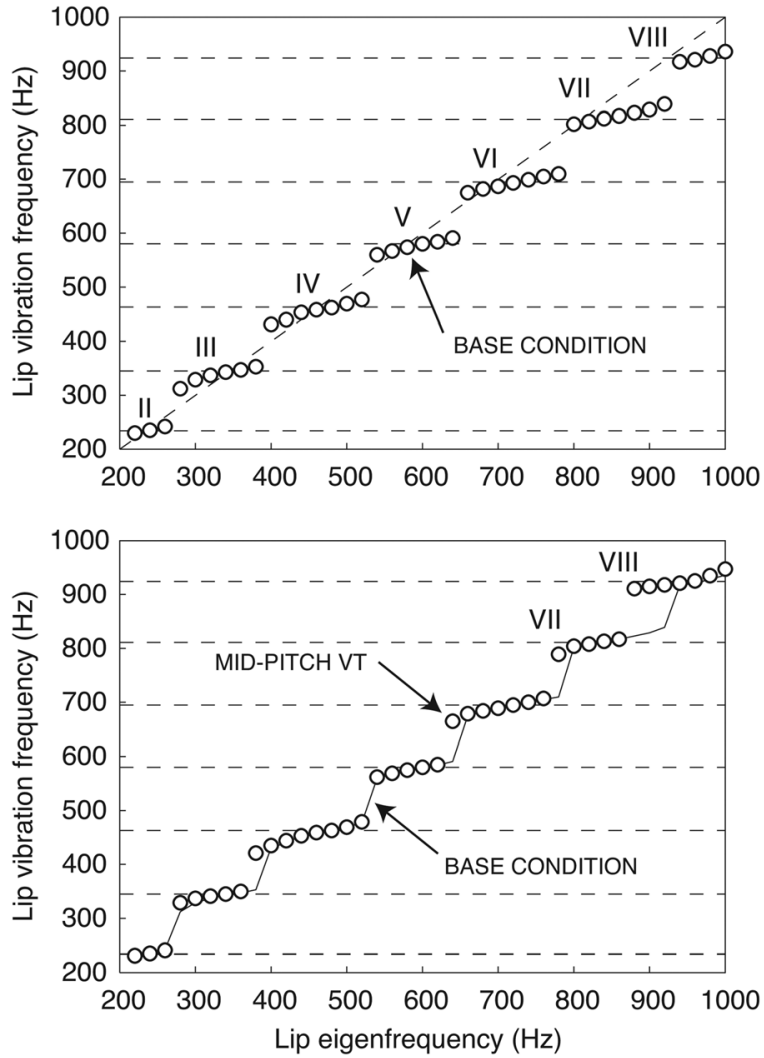

movement of the lips. The result for the low-pitch condition is plotted at the upper right of the figure. In this simulation, the input impedance of the player's air column was set using the low-pitch area function data irrespective of the eigenfrequency. Owing to the incorporation of the vocal-tract load, there is a mode jump at a lower eigenfrequency above the third mode, but the difference from the base condition is very slight. The results were similar under the mid-pitch vocal-tract condition shown at lower left, although there was a pitch jump to the eighth mode at a lower eigenfrequency than under the low-pitch condition.

Under the high-pitch condition plotted at lower right, the effect of the vocal tract is more noticeable than under the low- and mid-pitch conditions for the seventh and eighth modes. Note that the blowing frequency under the high-pitch condition was about $700 \mathrm{~Hz}$ in the MRI measurement, and the acoustic contribution of the vocal tract to the total impedance is apparent for frequencies greater than $700 \mathrm{~Hz}$ (see Figs. 5 and 6). In particular, the phase response in this frequency range is quite different from that for the low- and mid-pitch conditions. It is noteworthy that the vibration frequency was almost equal to the eigenfrequency when the eigenfrequency was higher than or equal to 940 Hz. Only under the high-pitch vocal-tract condition, the vibration frequency reached the ninth mode at an eigenfrequency of $1000 \mathrm{~Hz}$, although this datum point is not shown in the figure.
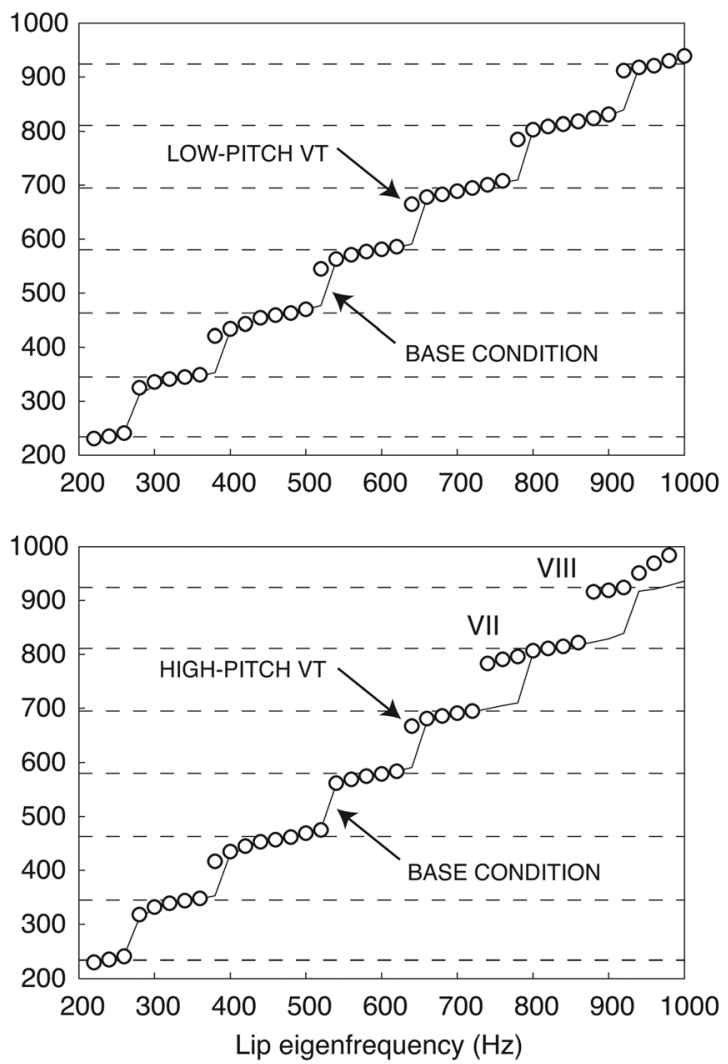

FIG. 8. Simulated lip vibration frequency as a function of the eigenfrequency. The acoustic feedback from the vocal tract was not considered in the base condition, and the resulting vibration frequency is plotted by circles in the upper left part. These data are connected by line segments at upper right, showing the results for the base condition as a reference. The circles are vibration frequencies obtained by setting the vocal-tract shape and hence the input impedance to the low-pitch condition. Similarly, the result for the mid-pitch condition is shown at lower left and the high-pitch condition at lower right. The broken, horizontal lines show the frequencies of the second to eighth impedance peaks. In this experiment, the blowing pressure was set to the values listed in Table I, so that a stable vibration of the lip was obtained. 


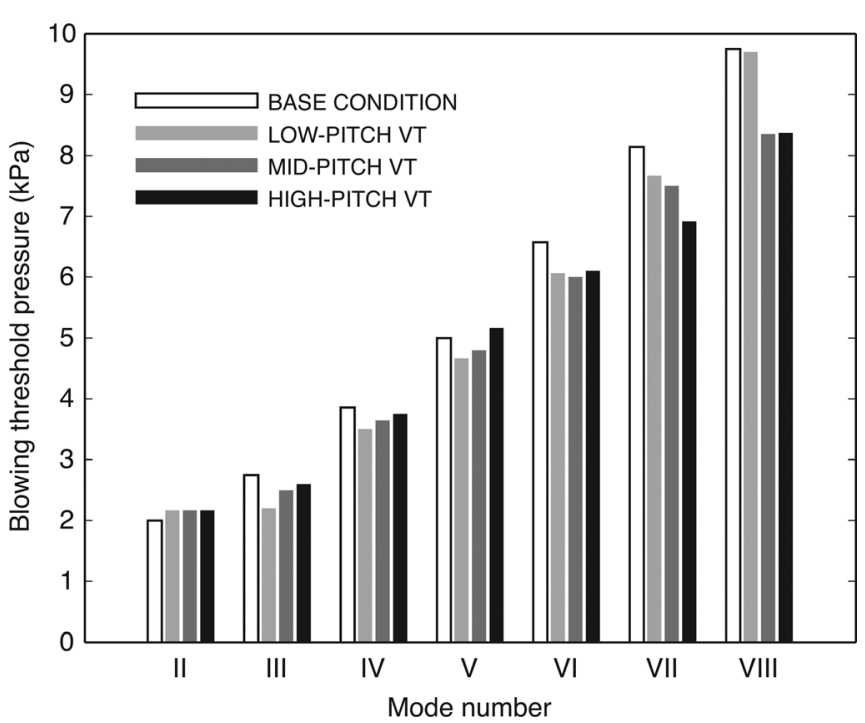

FIG. 9. Comparison of the blowing threshold pressure among the base and three vocal-tract conditions corresponding to low, mid, and high pitches. The pressure value was averaged for each mode number. The data samples used in this averaging were determined from the circles plotted in Fig. 8. For example, the sample number for the base condition was three for the second mode, six for the third, seven for the fourth, and so on. Three data points on the right-hand side of the high-pitch condition were excluded from the averaging; thus, the sample number for the eighth mode was three.

These results under the high-pitch condition can be explained using the regeneration theory. ${ }^{11,26}$ For frequencies $740-800 \mathrm{~Hz}$, the phase of the total impedance $Z\left(=Z_{\mathrm{trp}}+Z_{\mathrm{vt}}\right)$ was almost positive (bottom plot in Fig. 6). Because the phase of the mobility function, $G(f)$, is negative at the resonant frequency of the lip, ${ }^{10}$ this phase property was favorable to satisfy the requirement $\angle G(f)+\angle Z(f)=0$ and brought the pitch jump to the seventh mode. The increase in the magnitude of $Z$ (bottom plot in Fig. 5) could also support the jump because the requirement $\sqrt{2 p_{0} / \rho}|G(f)||Z(f)| \geq 1$ was more readily satisfied. For frequencies $880-900 \mathrm{~Hz}$, the phase of $Z$ was not completely positive but closer to zero by including the player's impedance in the total impedance, causing the jump to the eighth mode.

Last, Fig. 9 shows the simulation result with regard to the minimum blowing pressure required to generate lip oscillation. The pressure value was averaged for each vocal-tract condition and for each mode number. The figure shows that the threshold pressure in general increased as the mode number increased. Except for the second mode, the threshold pressure decreased when the vocal-tract load was incorporated in the simulation. It is also noteworthy that the pressure value under the low-pitch vocal-tract condition was the smallest among the four conditions for each of the third, fourth, and fifth modes. For the seventh and eighth modes, on the other hand, the high-pitch vocal tract had the effect of significantly reducing the blowing pressure more than $1 \mathrm{kPa}$ relative to the base condition.

This result can partly be explained as follows. From the condition $\sqrt{2 p_{0} / \rho}|G(f)||Z(f)| \geq 1$, the magnitude of the total impedance should be increased to compensate for a reduction in the blowing pressure, $p_{0}$. For the high-pitch vocal-tract condition, Fig. 8 shows that the seventh and eighth modes occurred at the lip eigenfrequency of 740-980 Hz, and it is shown in Fig. 5 that $|Z(f)|$ was increased by adding the player's impedance in this frequency range. On the other hand, for the eighth mode under the mid-pitch vocal-tract condition, the magnitude of the total impedance did not change clearly in the frequency range $880-1000 \mathrm{~Hz}$ even when the player's impedance was added. At this point, therefore, the regeneration theory is not enough to explain the simulation result.

\section{SUMMARY AND DISCUSSION}

The MRI study for a professional trumpet player showed that the tongue rose toward the palate going from low to medium to high pitch. Only limited morphological data have been recorded thus far for brass and woodwind players. Clinch et al. ${ }^{28}$ employed x-rays to show that a clarinet player adjusts the tongue in a similar manner as we observed. They also reported a change in the larynx position, which can be correlated with the tongue posture because they are connected by muscles and ligaments via the hyoid bone. The rising of the larynx has the effect of reducing the length of the vocal tract and increasing the resonance frequency, but no systematic change in the larynx position was found in our study.

Next, the area function of the vocal tract was estimated, and the input impedance of the player's air column was calculated. There were a number of peaks below $1 \mathrm{kHz}$, but the magnitudes of these peaks were smaller than those of the trumpet bore, indicating that the vocal tract has less effect in agreement with the foregoing study for the woodwind instrument. ${ }^{13}$ The total impedance including the instrument and player was also calculated, and changes in the phase response were observed according to the vocal-tract shape, especially when the tongue was raised under the high-pitch condition. Chen et al. ${ }^{15}$ mentioned that adjustment of the vocal-tract can affect the magnitude of the total impedance in pitch bending, but less influence was found in our study.

Backus, ${ }^{13}$ Chen et al., ${ }^{15}$ and Scavone et al. ${ }^{14}$ carried out acoustic measurements for the clarinet and saxophone to determine the effect of the vocal tract on the pressure in the vicinity of the mouthpiece. For brass instruments, however, such measurement is difficult for the vibrating lips, and therefore, computational models are useful. ${ }^{2,9,10,29}$ In our simulation, the vibration frequency of the lips was not so affected by the change in the vocal-tract shape, but with the vocal tract, the vibrating frequency shifted to a higher mode at a lower eigenfrequency, especially for the seventh and eighth modes of the instrument. This implies that the player can produce a high-pitch sound more easily with lower tension of the lips.

In addition, the vocal tract had a clear influence on the minimum blowing pressure required to attain the vibration of the lips. The numerical results show that to generate a lowpitch sound, the tongue should take a low position, which was typically observed when the player generated a low-pitch sound, to reduce the lung pressure effectively. To blow a high-pitch sound, on the contrary, the tongue should take a high position, which was typically observed when the player generated a high-pitch sound. Computer simulations suggested that the vocal tract can improve the efficiency of the performance in terms of the mode shift and required lung 
pressure, but the results obtained were for one specific player. The study will therefore be extended by increasing the number of players and vocal-tract shapes for various musical pitches to obtain a more general result.

\section{ACKNOWLEDGMENT}

This research was partly supported by the Grant-in-Aid for Scientific Research from the Japan Society for the Promotion of Science (Grant No. 19103003).

${ }^{1}$ J. Backus, "Input impedance curves for the brass instruments," J. Acoust. Soc. Am. 60, 470-480 (1976).

${ }^{2}$ R. Msallam, S. Dequidt, R. Caussé, and S. Tassart, "Physical model of the trombone including nonlinear effects. Application to the sound synthesis of loud tones," Acta Acustica 86, 725-736 (2000).

${ }^{3}$ M. W. Thompson and W. J. Strong, "Inclusion of wave steepening in a frequency-domain model of trombone sound production," J. Acoust. Soc. Am. 110, 556-562 (2001).

${ }^{4}$ L. Menguy and J. Gilbert, "Weakly nonlinear gas oscillations in air-filled tubes; solutions and experiments," Acta Acustica 86, 798-810 (2000).

${ }^{5}$ N. H. Fletcher, "Autonomous vibration of simple pressure-controlled valves in gas flows," J. Acoust. Soc. Am. 93, 2172-2180 (1993).

${ }^{6}$ S. Yoshikawa, "Acoustical behavior of brass player's lips," J. Acoust. Soc. Am. 97, 1929-1939 (1995).

${ }^{7}$ D. C. Copley and W. J. Strong, "A stroboscopic study of lip vibrations in a trombone," J. Acoust. Soc. Am. 99, 1219-1226 (1996).

${ }^{8}$ F.-C. Chen and G. Weinreich, "Nature of the lip reed," J. Acoust. Soc. Am. 99, 1227-1233 (1996)

${ }^{9}$ S. Adachi and M. Sato, "Time-domain simulation of sound production in the brass instrument," J. Acoust. Soc. Am. 97, 3850-3861 (1995).

${ }^{10} \mathrm{~S}$. Adachi and M. Sato, "Trumpet sound simulation using a two-dimensional lip vibration model," J. Acoust. Soc. Am. 99, 1200-1209 (1996).

${ }^{11}$ S. J. Elliott and J. M. Bowsher, "Regeneration in brass wind instruments," J. Sound Vib. 83, 181-217 (1982).

${ }^{12} \mathrm{~N}$. H. Fletcher and A. Tarnopolsky, "Blowing pressure, power, and spectrum in trumpet playing," J. Acoust. Soc. Am. 105, 874-881 (1999).

${ }^{13} \mathrm{~J}$. Backus, "The effect of the player's vocal tract on woodwind instrument tone," J. Acoust. Soc. Am. 78, 17-20 (1985).
${ }^{14}$ G. P. Scavone, A. Lefebvre, and A. R. da Silva, "Measurement of vocaltract influence during saxophone performance," J. Acoust. Soc. Am. 123, 2391-2400 (2008).

${ }^{15}$ J.-M. Chen, J. Smith, and J. Wolfe, "Pitch bending and glissandi on the clarinet: Roles of the vocal tract and partial tone hole closure," J. Acoust. Soc. Am. 126, 1511-1520 (2009).

${ }^{16}$ L. C. Merriman and J. A. Meidt, "A cinefluorographic investigation of brass instrument performance,” J. Res. Music Ed. 16, 31-38 (1968).

${ }^{17}$ J. Wolfe, J.-M. Chen, and J. Smith, "The acoustics of wind instrumentsand of the musicians who play them," in Proceedings of the 20th International Congress of Acoustics (ICA 2010), p. 1036.

${ }^{18}$ M. M. Sondhi and J. Schroeter, "A hybrid time-frequency domain articulatory speech synthesizer," IEEE Trans. Acoust., Speech, Signal Process. 35, 955-967 (1987).

${ }^{19}$ R. J. Baken and R. F. Orlikoff, Clinical Measurement of Speech and Voice, 2nd ed. (Singular Pub. Group, San Diego, 1999), pp. 517-524.

${ }^{20}$ B. H. Story, I. R. Titze, and E. A. Hoffman, "Vocal tract area function from magnetic resonance imaging," J. Acoust. Soc. Am. 100, 537-554 (1996).

${ }^{21}$ H. Takemoto, T. Kitamura, H. Nishimoto, and K. Honda, "A method of tooth superposition on MRI data for accurate measurement of vocal tract shape and dimensions," Acoust. Sci. Tech. 25, 468-474 (2004).

${ }^{22}$ K. Ishizaka, M. Matsudaira, and T. Kaneko, "Input acoustic-impedance measurement of the subglottal system," J. Acoust. Soc. Am. 60, 190-197 (1976).

${ }^{23}$ E. R. Weibel, Morphometry of the Human Lung (Springer-Verlag, Berlin, 1963), pp. 136-140.

${ }^{24}$ van den Berg, "An electrical analogue of the trachea, lungs and tissues," Acta Physiol. Pharmacol. Neerl. 9, 361-385 (1960).

${ }^{25}$ I. R. Titze, "Nonlinear source-filter coupling in phonation: Theory," J. Acoust. Soc. Am. 123, 2733-2749 (2008).

${ }^{26} \mathrm{~N}$. H. Fletcher, "Excitation in woodwind and brass instruments," Acoustica 43, 63-72 (1979).

${ }^{27}$ R. Caussé, J. Kergomrd, and X. Lurton, "Input impedance of brass musical instruments-comparison experiment and numerical models," J. Acoust. Soc. Am. 75, 241-254 (1984).

${ }^{28}$ P. G. Clinch, G. J. Troup, and L. Harris, "The importance of vocal tract resonance in clarinet and saxophone performance, A preliminary account," Acustica 50, 280-284 (1982).

${ }^{29}$ S. D. Sommerfeldt and W. J. Strong, "Simulation of a player-clarinet system," J. Acoust. Soc. Am. 83, 1908-1918 (1988). 